\title{
Ankle Joint Anterior Flexor Tendons
}

National Cancer Institute

\section{Source}

National Cancer Institute. Ankle Joint Anterior Flexor Tendons. NCI Thesaurus. Code C161389.

The tendons in the anterior compartment of the leg that connect muscles that originate on the surfaces of the femur, tibia, and fibula to bones in the toes and enable plantar flexion of the foot at the ankle. 